\title{
Brightness and loudness as functions of stimulus duration
}

\author{
JOSEPH C. STEVENS AND JAMES W. HALL \\ LABORATORY OF PSYCHOPHYSICS, HARVARD UNIVERSITY
}

The brightness of white light and the loudness of white noise were measured by magnitude estimation for sets of stimuli that varied in intensity and duration. Brightness and loudness both grow as power functions of duration up to a critical duration, beyond which apparent magnitude is essentially independent of duration. For brightness, the critical duration decreases with increasing intensity, but for loudness the critical duration is nearly constant at about $150 \mathrm{msec}$. Loudness and brightness also grow as power functions of intensity. The loudness exponent is the same for all durations, but the brightness exponent is about half again as large for short durations as for long. The psychophysical power functions were used to generate equal-loudness and equal-brightness functions, which specify the combinations of intensity $E$ and duration $T$ that produce the same apparent magnitude. Below the critical duration ET equals $k$ for equal brightness, and ET ${ }^{a}$ equals $k$ for equal loudness. The value $a$ is about 0.7 for threshold and about 1.25 for supraliminal loudness.

The present paper concerns the way apparent brightness and apparent loudness vary with duration throughout the dynamic range of vision and hearing. Interesting similarities and differences between these two senses come to light from a comparative study of brightness and loudness over wide variations of time and intensity.

The principal method was to obtain magnitude estimates of a matrix of stimuli covering a wide range of intensity ( $50 \mathrm{~dB}$ for brightness, $73 \mathrm{~dB}$ for loudness) and of duration (2000-fold for brightness, 100-fold for loudness). From the numerical estimates of a group of observers it is possible (1) to state how brightness and loudness grow with duration and with intensity, and (2) to generate a family of equal-sensation functions that specify combinations of duration and intensity that arouse a given level of apparent brightness or loudness.

Except for certain procedural details, the investigation of visual brightness was a repetition, with similar outcome, of an earlier experiment by Raab (1962). The range of intensity was extended 10 -fold $(10 \mathrm{~dB})$ in the present study. Aiba and S. S. Stevens (1964) used the method of brightness matching in order to study the same problem, and S. S. Stevens (1966b) has proposed a simple model to show how brightness may depend on intensity and duration.

An analogous model for loudness has not, apparently, been worked out. Data on supraliminal loudness are not very numerous, and there appear to be some puzzling discrepancies. Of most relevance to the present study are the equal-loudness functions measured by Miller
(1948) and by Small, Brandt, and Cox (1962) for white noise. White noise provides a more suitable stimulus for this kind of problem than pure tones, because the intensity of a white noise (unlike tones) can be rapidly modulated without effecting a material change in the sound spectrum. Thus a prominent click is heard at the onset of a tone but not at the onset of a noise.

How the loudness of white noise grows with intensity has been the subject of several investigations (S. S. Stevens, 1966a). Like brightness, the loudness of white noise obeys the general psychophysical power law proposed by S. S. Stevens (1961)

$$
\psi=k \phi .
$$

where $\psi$ is subjective magnitude, and $\phi$ is the physical magnitude. Of special concern here is how the constants of the power function depend on the duration of the stimulus.

\section{Apparatus for Producing Visual Flashes}

The flash source was a fast-decaying 4-watt fluorescent lamp (Sylvania's "Deluxe Cool White") that could be activated at a high intensity level for almost any duration longer than about $0.5 \mathrm{msec}$. The operation and circuitry are described by Gerbrands and J. C. Stevens (1964). Reasonably square pulses of light can be produced at durations as short as $0.5 \mathrm{msec}$. and at luminances as intense as 5 or 6 lamberts (L), about $107 \mathrm{~dB}$ re $10^{-10} \mathrm{~L}$.

From a distance of $35 \mathrm{~cm}$ the observer viewed foveally a portion of the lamp (diameter $1.25 \mathrm{~cm}$, visual angle $2^{\circ} 3^{\prime}$ ) from inside a dark booth (see Fig. 1). On a signal from the experimenter the observer pressed a key, which, after a delay of 0.5 sec., triggered the flash. The brief delay between pressing and seeing was found to favor concentration on the flash. Just to the left of the target appeared a red fixation spot. Throughout the experimental session the observer viewed this spot with his right eye while holding his head steady in a chin rest. A black mask prevented his left eye from seeing the target.

The intensity of the flash was controlled by means of neutral density filters.

\section{Procedure for Scaling Brightness}

Before an experimental session, the observer was dark-adapted with red goggles for $10 \mathrm{~min}$., and then for another minute or two he sat in the dark booth.

The observers were instructed to assign numbers proportional to the subjective brightness of the flashes 
and to disregard any apparent variation in the duration of the flashes. For the first flash presented to him, the observer was asked to decide on an appropriate number to stand for the subjective brightness; to all subsequent stimuli he then assigned numbers proportional to subjective brightness.

Altogether, 60 stimuli (i.e., combinations of peak luminance and duration) were presented in a series of six experiments (see Table 1). Approximately one-sixth of these stimuli were used in each of the six experiments, but in order to tie the results of all six experiments together, one particular stimulus $(200 \mathrm{msec}$. at $85 \mathrm{~dB}$ re $10^{-10} \mathrm{~L}$ ) was presented in every experiment. The sequence of the various experiments differed from one observer to another.

Each stimulus was presented twice in an experimental session, except that the first stimulus was presented three times with only the last two judgments counted. This procedure was adopted because extensive experience with the method of magnitude estimation has shown that the original number assigned as a "modulus," whether by the experimenter or by the observer himself, is not always exactly the same as the "effective" modulus implicit in the subsequent judgments.

The order of the stimuli was irregular with respect to brightness and differed from one observer to another. The dimmest stimulus was never presented immediately after the brightest, and all the stimuli were presented once before any was presented a second time. The time between presentations was about $15 \mathrm{sec}$. If an observer felt that he had not seen a given flash properly, he could ask for another presentation.

Altogether 21 persons (graduate students, staff members, and employees) served as the observers. Some of them served in only one experiment, others in $2,3,4$, 5 , or all 6 of the experiments, but 10 persons served in each of the six experiments. No person ever served in more than one experiment on the same day.

\section{Apparatus for Producing Bursts of Noise}

An electronic switch (Grason-Stadler Type 829-S-14) was used in conjunction with an interval timer (GrasonStadler Type 471) to gate the filtered output (low-pass $20,000 \mathrm{~Hz}$ ) of a white noise generator. The gated signals were then passed through a decade attenuator to a pair of PDR-8 earphones. The duration and the intensity of the bursts were monitored throughout the experiment with an oscilloscope and a voltmeter.

The interval timer was set to deliver repeated bursts of noise, separated by $900-$ msec. intervals of silence. On a given stimulus presentation the observer pressed a switch for as long as he wished to hear the repeated bursts. The observer sat in a sound isolation booth and listened binaurally.

\section{Procedure for Scaling Loudness}

The task was to make magnitude estimations of apparent loudness, disregarding any other perceived

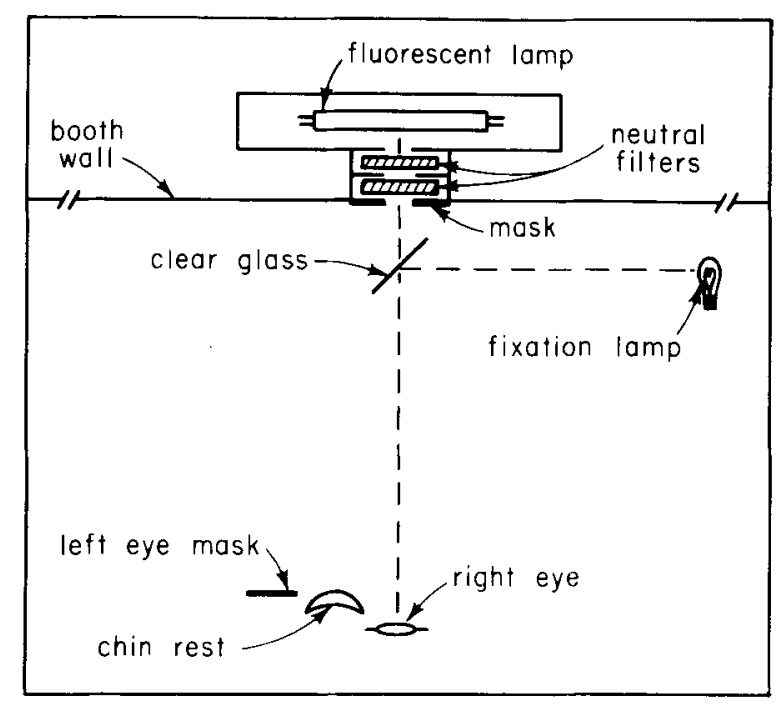

Fig. 1. Schematic diagram of the visual apparatus (not to scale).

variation, such as duration or quality (qualitative variation as a function of duration constitutes a prominent feature of the shorter bursts). Sixty combinations of physical magnitude (peak SPL) and duration were presented in a series of six experiments as specified by Table 2 . One stimulus, $80 \mathrm{msec}$. at $81 \mathrm{~dB}$ peak SPL, was presented in each of the six experiments. The order of these experiments differed from one observer to another.

Each stimulus was presented twice in a session, except for the first stimulus (always one of intermediate loudness), which was presented three times altogether; only the last two judgments of this stimulus were counted as magnitude estimates. In each session the order of the stimuli was irregular with respect to loudness, except that all the stimuli were presented once before any was presented a second time. The order of stimuli also differed from one observer to another.

Twelve persons served as observers in all six experiments. Ten of them were undergraduate students, one a graduate student, and one a staff member. Before the first session the observers were asked to make magnitude estimates of the length of a few line segments in order to given them familiarity with the method and

Table 1. The combination of physical magnitude and duration used as stimuli in Experiments 1 through 6 on brightness. For Experiments 1 to 3 , the first stimulus was $200 \mathrm{msec}, 85 \mathrm{~dB}$. The asterisks mark the first stimuli for Experiments 4 to 6 .

\begin{tabular}{lllllllllllll} 
Peak luminance & \multicolumn{10}{c}{ Duration in milliseconds } \\
in dB re $10^{-10} \mathrm{~L}$ & 0.5 & 1 & 2 & 5 & 10 & 20 & 50 & 100 & 200 & 500 & 1000 \\
\hline 105 & 2 & 4 & 3 & 2 & 5 & 3 & 2 & 4 & 3 & 2 & 3 \\
95 & 5 & 6 & 4 & 5 & 6 & 4 & 5 & 6 & 4 & 5 & 6 \\
85 & 2 & 1 & 3 & 2 & 1 & 3 & 2 & 1 & $A L L$ & 2 & 1 \\
75 & 5 & 6 & 4 & 5 & 6 & 4 & 5 & 6 & $4^{*}$ & $5^{*}$ & $6^{*}$ \\
65 & & 1 & 3 & 2 & 1 & 3 & 2 & 1 & 3 & 2 & 1 \\
55 & & & & & & 4 & 5 & 6 & 4 & 5 & 6 \\
\hline
\end{tabular}


Table 2. The combination of physical magnitude and duration used as stimuli in Experiments 1 through 6 on loudness.

\begin{tabular}{lllllllllll} 
Peak SPL in $\mathrm{dB}$ re & \multicolumn{10}{c}{ Duration in milliseconds } \\
0.0002 dyne $/ \mathrm{cm}^{2}$ & 5 & 10 & 18 & 30 & 50 & 80 & 130 & 200 & 300 & 500 \\
\hline 36 & 2 & 4 & 6 & 4 & 2 & 6 & 4 & 6 & 2 & 6 \\
51 & 3 & 2 & 4 & 5 & 4 & 2 & 6 & 1 & 6 & 2 \\
66 & 6 & 5 & 2 & 1 & 4 & 3 & 2 & 4 & 5 & 3 \\
81 & 5 & 6 & 1 & 2 & 3 & $\mathrm{ALL}$ & 1 & 3 & 5 & 6 \\
96 & 4 & 1 & 3 & 5 & 6 & 1 & 3 & 4 & 2 & 1 \\
109 & 1 & 3 & 5 & 3 & 5 & 3 & 1 & 5 & 1 & 5 \\
\hline
\end{tabular}

to improve their understanding of the task. No person served in more than one session in one day (typically, sessions were separated by several days), and none of the observers had previously served in any of the six experiments on brightness.

\section{Measurement of the Auditory Threshold}

The absolute threshold of a dozen observers (only one of whom served in the scaling experiment) was measured at each of the 10 durations listed in Table 2 and also at durations of 800 and $1000 \mathrm{msec}$. The order of presentation of the durations was irregular and different for each observer.

For this experiment a recording attenuator (GrasonStadler Model E-3262-A) was inserted between the electronic switch and the earphones. The observer operated a two-position switch, which when pressed caused an immediate 1-dB increase or 1-dB decrease of attenuation in the recording attenuator. A light flash signaled the observer that the noise burst was about to occur. The task was to press the switch in one direction (increased attenuation) if the noise was heard and in the other direction (decreased attenuation) if the noise was not heard. The threshold was "tracked" by this staircase method (the Békésy method) until a reasonably stable value was achieved.

The observer practiced the technique for about 10 min. After a rest of two or more hours, he returned for the actual experimental session, which lasted 30 to $40 \mathrm{~min}$.

\section{Results of Scaling Brightiess}

For each stimulus the geometric mean of the brightness estimates was computed. In order to tie together the results from the six different experiments, the following procedure was adopted. For each experiment, the geometric mean for the stimulus common to all six experiments (85 dB, 200 msec.) was multiplied by a factor $b$ that made the resulting product (b times the geometric mean) equal 10 . Then all the geometric means for a given experiment were multiplied by the same factor $b$. This computation preserves the ratios among the geometric means of a given experiment and allows the results of all six experiments to be plotted in a single pair of coordinates.

The transformed geometric means are plotted as functions of duration in the log-log coordinates of Fig. 2. The parameter is peak luminance in $\mathrm{dB}$ re $10^{-10} \mathrm{~L}$. Three features of the outcome, all of which have been observed in earlier studies (Aiba \& S. S.Stevens, 1964; Raab, 1962) may be noted again here. (1) For each intensity level there is a "critical duration," shorter than which apparent brightness clearly depends on the duration, and longer than which brightness is essentially independent of the duration. Below the critical duration, the data can be fitted fairly well by straight lines whose slopes equal approximately 0.45. In other words, to a first approximation the brightness grows as a power function whose exponent is about 0.45 . This exponent is larger than the exponent of the "standard" bril function $(0.33)$ that relates subjective brightness to the luminance of a target that lasts $1 \mathrm{sec}$. and is viewed by the darkadapted eye (J. C. Stevens \& S. S. Stevens, 1963). (2) In the region of the critical duration, there usually occurs an enhancement (shown by the dotted "humps" in Fig. 2),

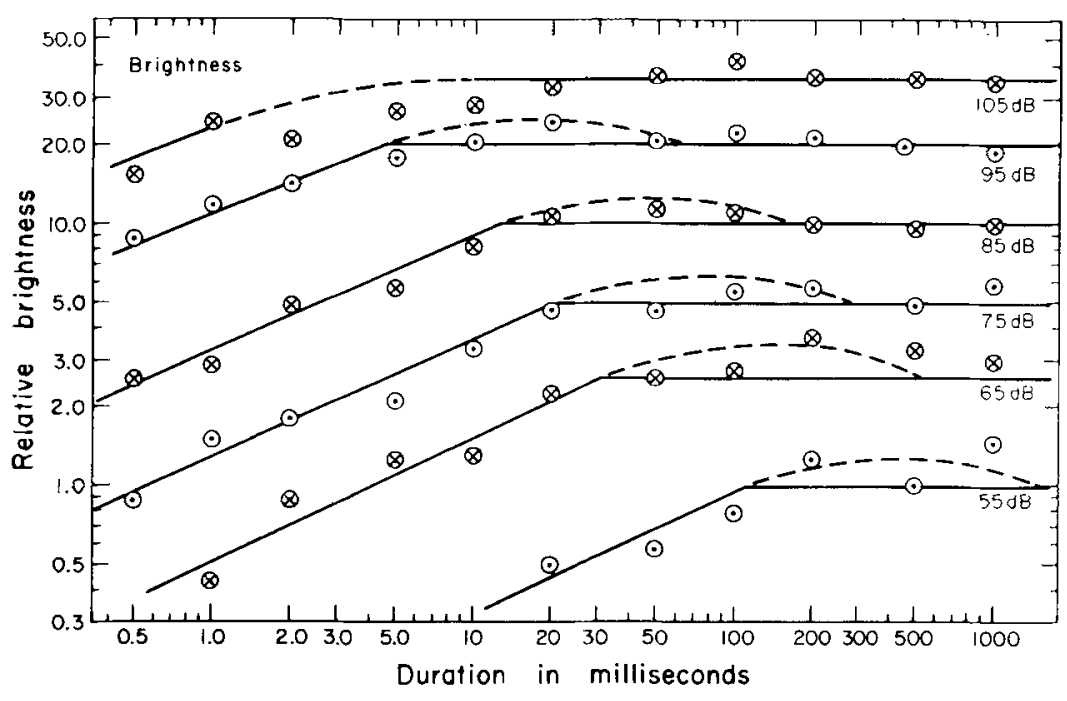

Fig. 2. How apparent brightness grows as a function of stimulus duration at six levels of peak luminance measured in dB re $10^{-10} \mathbf{L}$. 
which is the well-known Broca-Sulzer phenomenon. For an unknown reason, a Broca-Sulzer hump failed to show up in the topmost function of Fig. 2 (105 dB). The flattened appearance of this function is puzzling. It resembles the topmost function in a similar plot of Raab's results (see S. S. Stevens, 1966b) at a level of $95 \mathrm{~dB}-\mathrm{a}$ level that revealed the enhancement in the present study. Why the topmost functions of these two different experiments should have the same curiously flattened shape is not clear, especially since a BrocaSulzer hump occurred in the brightness matches of Aiba and S. S. Stevens (1964) at a level of $103 \mathrm{~dB}$. (3) The critical duration decreases with the intensity level, from about $150 \mathrm{msec}$. at $55 \mathrm{~dB}$ to about $5 \mathrm{msec}$. at 95 dB. This decrease in the critical duration was present but had a smaller magnitude in the matches of Aiba and S. S. Stevens than in either Raab's magnitude estimations or those of the present study.

\section{Brightness as a Function of Intensity}

If, for the longer durations, apparent brightness grows as a power function of luminance, then the horizontal portions of the functions of Fig. 2 ought to be spaced at equal intervals in these log-log coordinates. This is approximately true, with the obvious exception of the lowest function (55 dB). Between 65 and $105 \mathrm{~dB}$, an increase of $10 \mathrm{~dB}$ produces nearly a constant two-fold increase in subjective brightness. Below $65 \mathrm{~dB}$, the growth of brightness seems to be steeper. Near the absolute threshold (roughly $50 \mathrm{~dB}$ for a $2^{\mathrm{O}}$ target), the growth of sensory magnitude with intensity is known to be rapid. The generality of this finding throughout the sensory domain has led to a more general form of the power law:

$$
\psi=\mathrm{k}\left(\phi-\phi_{\mathrm{o}}\right)^{\beta}
$$

where $\phi_{0}$ approximates the absolute threshold (Ekman,

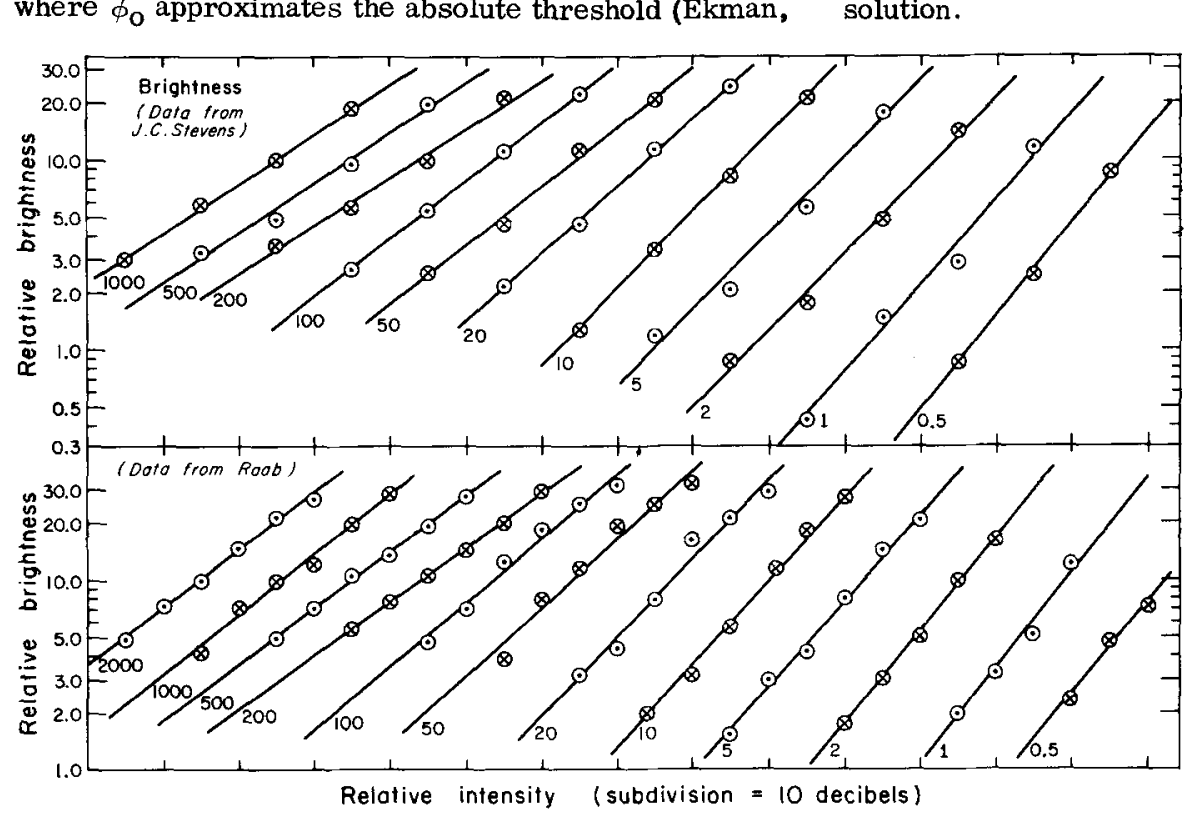

1958; Luce, 1959; J. C. Stevens \& S. S. Stevens, 1961). The larger space between the functions for 55 and $65 \mathrm{~dB}$ is consistent with Equation (2).

Figure 2 seems to imply that the growth of brightness with intensity follows the same power law fordurations longer than about $100 \mathrm{msec}$. Except for the function at $105 \mathrm{~dB}$, it would also appear that a power function may hold for durations shorter than about $5 \mathrm{msec}$. The spacing of the functions is such that, as suggested by Aiba and S. S. Stevens (1964), the exponent for short durations must be larger than that for the longer durations. Because the critical duration varies with intensity between about 10 and $100 \mathrm{msec}$, the brightness functions should consist of two sections, the upper portion of which would be steeper than the lower.

The data are displayed in another way in Fig. 3, where the adjusted geometric means for the stimuli between 65 and $95 \mathrm{~dB}$ are plotted as a function of peak luminance, with duration as the parameter. Also plotted in Fig. 3 are the geometric means obtained by Raab (see S. S. Stevens, 1966b) for peak luminance between 65 and 90 $\mathrm{dB}$ and for a dozen durations between 0.5 and $2000 \mathrm{msec}$. Although the data for intermediate durations could probably be fitted better with two straight-line segments, power functions were fitted by least squares to the data for all durations. The obtained slopes (exponents) were plotted as circles and squares in Fig. 4. The two sets of exponents follow the same trend, but Raab's exponents are slightly larger throughout. The exponents range from about 0.3 for the longer durations to about 0.45 for the shorter durations. The transition from the one exponent to the other for the intermediate durations appears to be gradual rather than abrupt; presumably this happens because with decreasing duration the steeper segment of the two-segment function comes more and more to dominate in the least-squares solution.

Fig. 3. How apparent brightness grows as a function of stimulus magnitude for a number of different stimulus durations (in milliseconds). Upper part: present experiment. Lower part: experiment by Raab (1962). The peak luminance of the most intense stimulus plotted was 95 $\mathrm{dB}$ (upper) and $90 \mathrm{~dB}$ (lower) re $10^{-10} \mathrm{~L}$. Lines fitted by the method of least squares. 


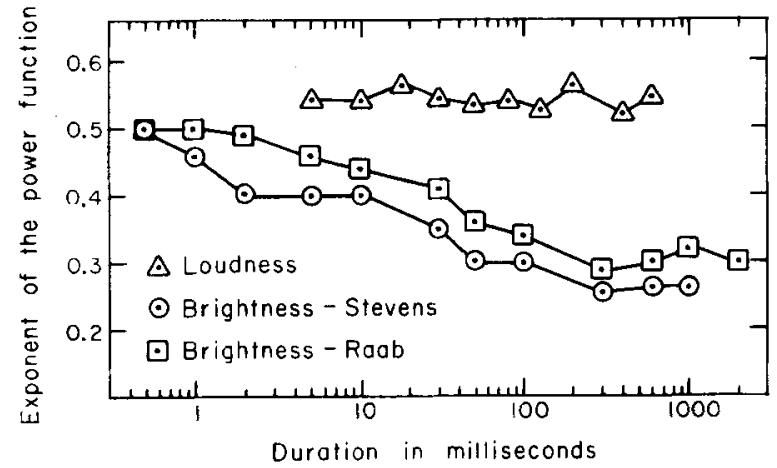

Fig. 4. How the exponents of the loudness and brightness functions shown in Figs. 3 and 7 depend upon the duration of the stimulus.

The exponent of the "standard" brightness function (the so-called bril scale) has been given a value of 0.33 , a value estimated from the results of a variety of methods in different laboratories (see S. S. Stevens, 1966b). It is not unusual, however, for the method of magnitude estimation to yield a value slightly smaller than 0.33 (J.C.Stevens \& S.S.Stevens, 1963). Moreover, the likelihood of obtaining a low exponent appears to increase when a task is made more difficult or complicated (Marks, in press).

Both sets of data in Fig. 4 suggest that the exponent is about one and a half times larger for short than for long flashes. If the exponent of the bril function is assumed to be 0.33 , the exponent for short flashes may be estimated to be close to 0.5 . These were the values for short and long flashes estimated earlier by S. S. Stevens (1966b) from Raab's data. The matches of Aiba and S. S. Stevens (1964) give a smaller estimate of the difference $(0.33$ and 0.40$)$.

Figure 4 also shows that, in contrast to brightness, the exponent of the loudness function (triangles) is essentially invariant with duration. This functional difference between the eye and the ear is discussed below.

\section{The Equal-8rightness Functions and Bloch's Law}

The straight lines of Fig. 3 were used to generate a family of equal-brightness functions that relate peak luminance and duration. Several horizontal cuts were made through the power functions of Fig. 3. The peak Iuminances in decibels and the durations in milliseconds marked by the intersections were plotted in Fig. 5, and straight-line segments were fitted to the points. This procedure does not eliminate the deficiencies in the data, nor does it preserve the Broca-Sulzer hump. On the other hand, it clarifies certain relations.

The dotted lines of Fig. 5 mark the "critical durations," longer than which brightness is essentially independent of duration. The equations of the dotted lines are very nearly the same for both sets of data in Fig. 5.

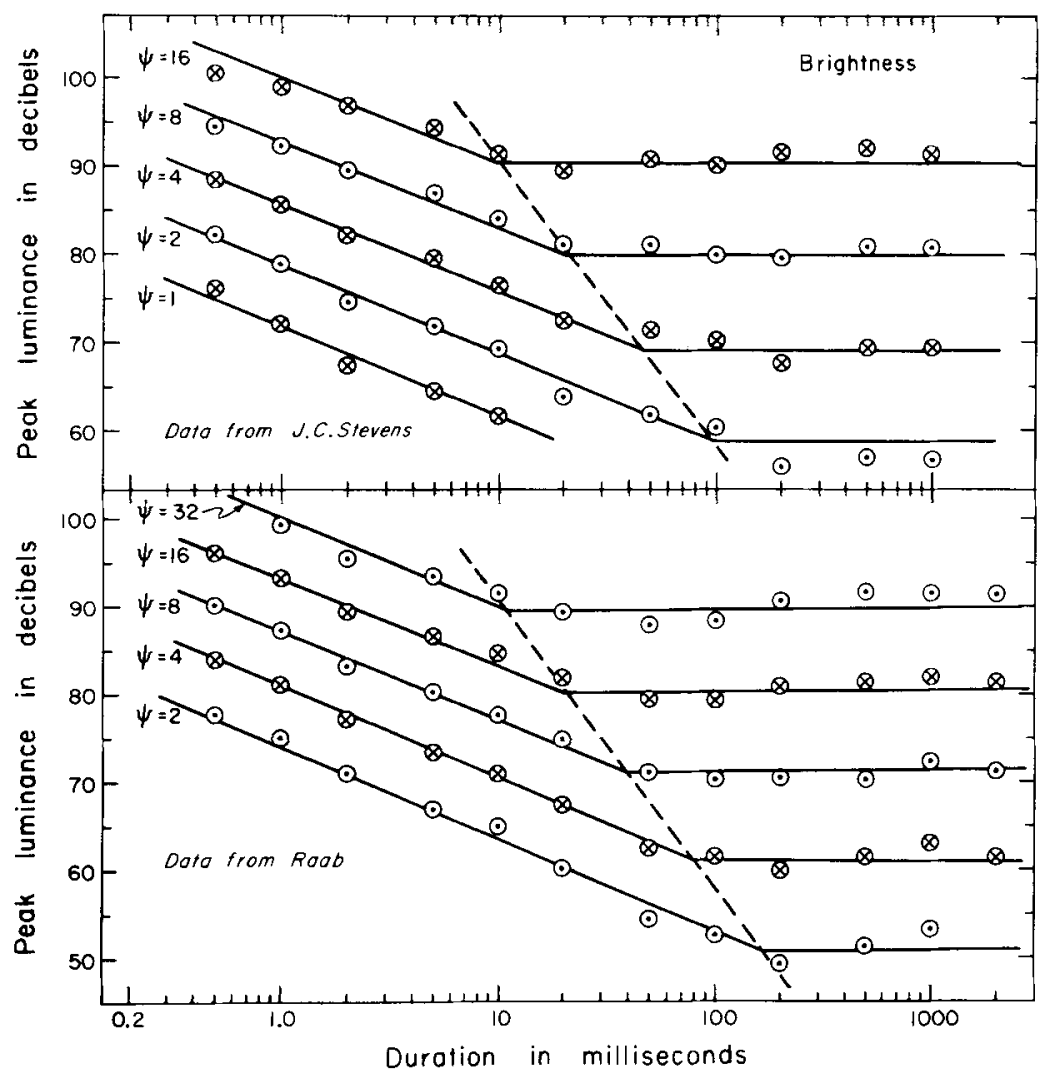

Fig. 5. Two families of equal-brightness functions giving the combinations of peak luminance and duration that produce the same apparent brightness. Upper: present experiment. Lower: experiment by Raab. The points were obtained by making horizontal cuts through the brightness functions of Fig. 3 at several brightness values $\psi$ 


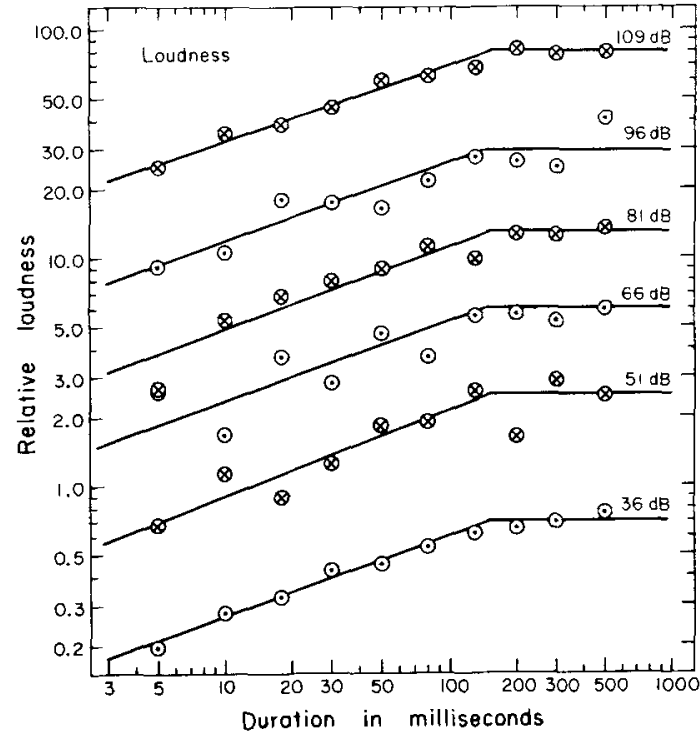

Fig. 6. How subjective loudness grows as a function of stimulus duration at six levels of stimulus magnitude (in d8 peak SPL).

The straight-line segments to the left of the dotted lines have slopes equal to -1.0 . This means that a decade increase in duration may be traded for a decade decrease in peak luminance, and vice versa. This relation of reciprocity between time and intensity, demonstrated in Fig. 5, means that Bloch's Law holds at suprathreshold levels, as noted by Aiba and Stevens and by Raab. On this point all three studies agree. Because time and intensity are interchangeable below the critical duration, the exponent that governs the power function relating subjective brightness to duration must have a value of 0.4 or 0.5 . Figure 2 shows that this conclusion is approximately correct.

\section{The Rise-Time of Visual Sensation}

The negative slope of the dotted lines in Fig. 5 suggests that the time it takes for the development of visual sensation depends on the level of stimulation. That the time required for maximum sensation grows shorter with increased intensity has been recognized by virtually every investigator of the problem. According to Bills (1920), various functions have been proposed to relate the "rise-time" to the intensity. Among these was Charpentier's interesting suggestion in 1887 that the time to maximum sensation varies inversely with the fourth root of intensity. McDougall's measurements in 1904 suggest the fifth root of intensity, and the dotted lines of Fig. 5 suggest the third root of intensity. Those lines have slopes (exponents) of -3.2 (upper line) and -3.3 (lower line).

The slope values in Fig. 5 raise the interesting possibility that the time required for maximum sensation may grow in simple inverse proportion to the subjective brightness that is finally attained; for if brightness grows as the cube root of intensity (the bril function) and if the reciprocal of duration at maximum brightness also grows as the cube root of intensity (as shown by the dotted lines), then it follows that the critical duration grows in inverse proportion to the maximum brightness. The equal-brightness contours of Aiba and S.S.Stevens, however, show a variation of the critical duration that is too small to obey the cube-root relation.

\section{Results of Scaling Loudness}

The loudness estimates were processed in the same way as were the brightness estimates. In order to tie together results from the six different experiments, the geometric means for each experiment were multiplied by a factor $b$; the value of the factor $b$ was chosen so that the product of $b$ and the geometric mean for the stimulus $81 \mathrm{~dB}$ for $80 \mathrm{msec}$. (the one presented in all six experiments) equalled 10. In Fig. 6 the 60 adjusted geometric means are plotted as a function of duration with peak SPL as the parameter. In Fig. 7 they are plotted as a function of peak SPL with duration as the

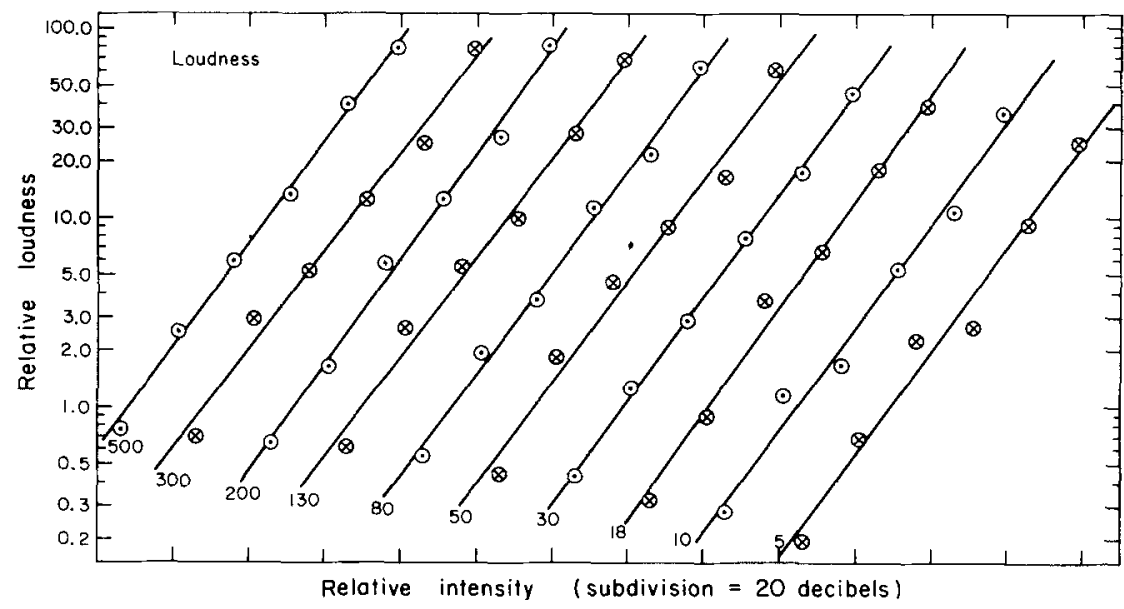

Fig. 7. How the subjective loudness of white noise grows as a function of stimulus magnitude for 10 different stimulus durations (in milliseconds). The most intense stimulus for each function was $\mathbf{1 0 9}$ dB peak SPL. Lines fitted by the method of least squares. 


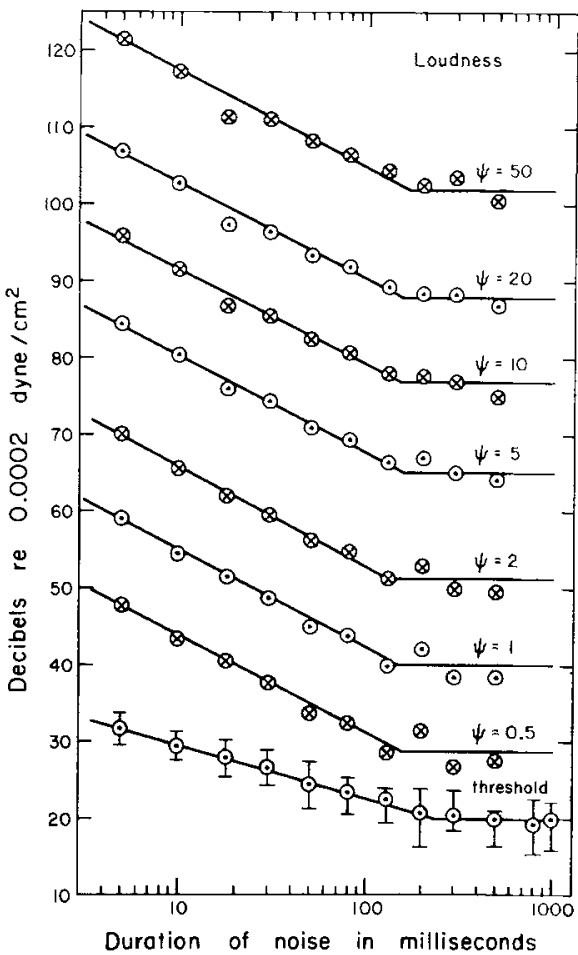

Fig. 8. Equal-loudness functions giving the combinations of duration and peak sound pressure level that produce the same subjective loudness. The points were obtained by making horizontal cuts through the functions of Fig. 7 at several levels of apparent loudness $\psi$. The lowest function shows how the threshold varies with duration; each point is the average of the data from $12 \mathrm{ob}-$ servers, and the vertical lines show the interquartile ranges.

parameter. In their own way each of these graphs spotlights certain aspects of the outcome.

Figure 8 shows that up to about $150 \mathrm{msec}$. the subjective loudness grows approximately as a power function of the duration of the noise. The straight-line segments have slopes in the vicinity of 0.35 . With further increase of duration the loudness grows little, if at all. The exponent 0.35 differs significantly from the exponent of the power function that governs the growth of loudness with stimulus magnitude (the so-called sone function). Typically, the exponent for loudness is 0.3 if the stimulus is measured in sound energy or, alternatively, 0.6 if the stimulus is measured in sound pressure. (Energy is proportional to the square of pressure.) In the present experiments the exponent for all durations falls a little below that of the sone function, as can be seen in Fig. 7 and in the plot of the exponents given in Fig. $4-0.27$ in terms of sound energy ( 0.54 in terms of sound pressure). As mentioned above, the method of magnitude estimation tends to yield a low exponent when a task is difficult. In the present experiment the difficulty of judgment was compounded because the noises differed in apparent quality as well as in apparent loudness and apparent duration. The point here, however, is not the absolute size of the exponent obtained but rather the difference between the exponents that govern the loudness functions for intensity and duration.

Figure 6 reveals no variation of the "critical duration" with the level of intensity nor anything resembling a Broca-Sulzer hump. Instead, the relation between loudness and duration seems to be about the same over a great portion of the dynamic range of hearing. This generalization needs qualification, however, when it comes to loudness near the absolute threshold.

The lines in Fig. 7 were fitted to the points by the method of least squares, and the slopes (exponents) are plotted in Fig. 4 to show that, unlike visual brightness, the loudness of a white noise seems to be governed by the same exponent regardless of the duration. The average of the obtained exponents was 0.54 and the standard deviation, 0.01 . The loudness functions of Fig. 7 seem to differ from one another with respect only to the constant k of Equation (1), that is to say, with respect to the stimulus magnitude that is required to produce a given level of loudness. Using bursts of tone at 1000 $\mathrm{Hz}$ instead of noise, Wright (1965) found the exponent of the loudness functions to be constant. His five observers estimated the loudness of a number of intensity levels at durations of $10,30,100,300$, and $500 \mathrm{msec}$.

\section{The Equal-Loudness Contours and the Critical Duration}

The functions of Fig. 7 were used to generate a family of equal-loudness functions relating peak SPL and duration-an auditory analogue of the family of of brightness functions pictured in Fig. 5. Several horizontal cuts were made through the functions of Fig. 7; the intensity in decibels and the duration in milliseconds marked by the intersections were then plotted in Fig. 8, and each function was represented by two straight-line segments.

Two main principles emerge from the family of functions. (1) The shape of the equal brightness functions is substantially invariant over the whole auditory range. Local perturbations that show up in several of the functions, such as the hump at $200 \mathrm{msec}$., probably owe their existence to the small random variations in the constants of the power functions in Fig. 7. (2) An increase in duration above $150 \mathrm{msec}$. brings about little, if any, change in loudness at any level of stimulus magnitude. Below $150 \mathrm{msec}$, however, a change in stimulus magnitude can offset a change in duration in order to preserve the same loudness. This trading relation does not appear, however, to obey the simple rule of reciprocity that governs visual brightness, because the line segments lack the required slope of -1.0. For example, to offset a 10-fold change in the duration requires a $12.5-\mathrm{dB}$ change in the stimulus magnitude (i.e., an 18-fold change in terms of sound energy or a 4.2-fold change in terms of sound pressure).

In a number of other studies using tones or noises, straight-line segments like those in Fig. 8 have been fitted to threshold functions and to equal-loudness functions obtained by the method of loudness matching. 
The fits are often good, although the various studies fail to yield precisely the same slope. For noise, Miller (1948) measured an average slope of -0.88 for three listeners (i.e., $-8.8 \mathrm{~dB}$ offsets a 10-fold change in duration), and Small, Brandt, and Cox (1962) measured an average slope of -1.25 for 12 listeners, the same slope obtained in the present experiment. Both Miller and Small et al stated that the critical duration depends on the level of intensity. The evidence offered for this dependency does not seem to be definitive, and, in any case, in neither study is the apparent variation as large as the variation measured for visual brightness. Over a range of intensities comparable to that used in the present experiment on loudness $(70 \mathrm{~dB})$, the critical duration in Miller's experiment appears to have changed by a factor of 1.5 or less. Small and his co-workers measured a change of approximately 3-fold between 20 and $60 \mathrm{~dB}$ above threshold.

The slope of -1.25 obtained here implies that subjective loudness grows less rapidly with respect to sound energy than with respect to duration. The formula relating peak energy $\mathrm{E}$ and duration $\mathrm{T}$ for equal loudness below the critical duration is

$$
\text { or } \quad \begin{array}{ll}
\log E=-1.25 \log T+\log C \\
E T^{1.25}=C
\end{array}
$$

Equation (4) implies that the exponent of the psychophysical power function should be 1.25 times larger for duration than for sound energy. A comparison of the slopes of the functions in Figs. 6 and 7 confirms this expectation.

\section{Is There a Crifical Duration for Loudness?}

Although the equal-loudness functions can be described quite well by two straight-line segments, the points in Fig. 8 could be fitted by other types of functions, such as a logarithmic or exponential curve. Several different formulas have from time to time been proposed to describe the relation between duration and intensity at the absolute threshold. Considering the nature and the precision of the psychophysical methods available for the determination of thresholds and equal loudness, it may not prove feasible to decide on the basis of fit alone from among all the alternative formulas and theories. Whether the critical duration should be thought of as a real discontinuity is therefore debatable; but it is nevertheless clear that a sharp change in the slope of the equal-loudness function does occur in the vicinity of $150 \mathrm{msec}$. Small, Brandt, and Cox reported that the critical duration differs widely from one listener to another and that the transition between the two segments of the functions is more gradual for the averaged results than it is for the individual results. Thus, the real sharpness of the change is partially smoothed over by the process of averaging.

However that may be, the equal-loudness contours of Fig. 8 provide a reasonable and simple description of the empirical findings. In Fig. 9 the values of the

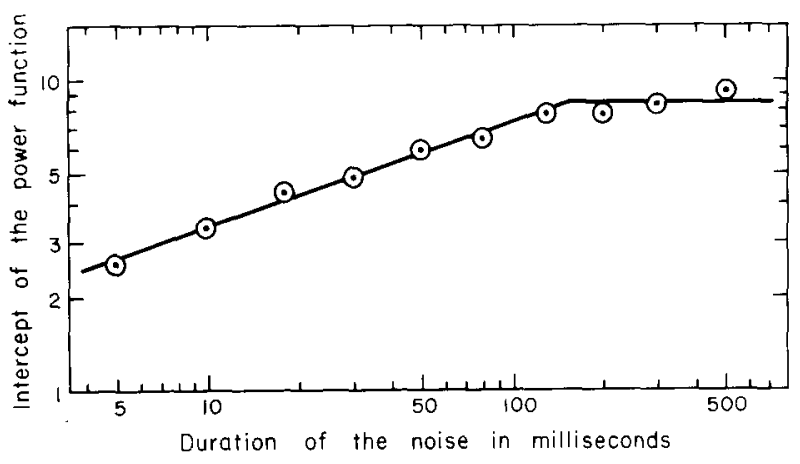

Fig. 9. The value of the constant $k$ of the psychophysical power function for loudness, plotted as a function of stimulus duration, in $\log -\log$ coordinates.

constant $\mathrm{k}$ of the power functions presented in Fig. 7 are plotted in log-log coordinates. The straight-line segments in Fig. 9 show that the value of $\mathrm{k}$ grows approximately as the 0.34 power of duration up to about $150 \mathrm{msec}$. and beyond $150 \mathrm{msec}$. remains approximately constant. If this model is correct, then the general formula for loudness (below the critical duration) can be written:

$$
L=c \operatorname{T} \alpha E \beta
$$

In the present experiment the values of $\alpha$ and $\beta$ were 0.34 and 0.272 , i.e., $\alpha$ is 1.24 times larger than $\beta$. Since the correct value of $\beta$ is thought to be about 0.3 (the sone function), Equation (5) can be rewritten:

$$
L=c T^{0.375} E^{0.3}
$$

Equation (4) for the equal-loudness contours follows directly from Equation (6).

\section{Reciprocity and Threshold}

The absolute threshold of audibility (average from 12 observers) is plotted as the bottom function of Fig. 8 . This function resembles the equal-loudness contours in its double-segmented shape, but it appears to differ from them in two ways. First, the critical duration at threshold is about $230 \mathrm{msec}$, rather than $150 \mathrm{msec}$. Thus, between about 0 and $10 \mathrm{~dB}$ sensation level (SL) a shift takes place in the critical duration, as is apparent also from the experiments of Miller and of Small, Brandt, and Cox. Second, the slope of the audibility function $(-0.7)$ is substantially different from that of the equal-loudness functions $(-1.25)$.

A slope of -0.7 agrees reasonably well with Miller, who obtained -0.8 , with Garner (1947), who obtained -0.88, and with Small, Brandt, and Cox, who obtained -0.77. Note that in that experiment Small et al also obtained a slope of -1.25 for equal-loudness contours between 10 and $60 \mathrm{~dB}$ SL. Thus the evidence is strong that stimulus magnitude and duration do not offset each other in the same way at threshold and at higher levels. At threshold, furthermore, the slope for noise does not 
equal -1.0. Over a similar range of duration, the slope for a pure tone may be closer to -1.0 , and nearly perfect energy summation may take place. Although reciprocity has often been claimed for pure tones, at least one extensive study of the question (Yntema, 1955) yielded results much closer to those reported here for noise. Garner (1947) pointed out that simple reciprocity for tones clearly breaks down, not only at the critical duration, but also toward very short durations, as the spectrum of the tone burst comes more and more to resemble that of noise; and he suggests that perfect temporal summation may occur only when the stimulus is confined to a "critical band." Broader spectra, such as broad-band noises or very short tones, yield less than perfect summation. Other interpretations of the same facts are, however, given by Zwislocki (1960), and the present experiments demonstrate that the degree of summation for noises depends on the intensity level.

In any case, the measurements of threshold and equal loudness alike seem to fit the formula suggested by Garner for relating time and energy, namely:

$$
E T^{a}=k
$$

It is curious that, for the same noise spectrum, the factor a should assume values both greater and smaller than 1.0, depending on the stimulus level. There seems to be no analogous phenomenon in vision. The change in the factor a between threshold and higher levels becomes, therefore, a fact to be fitted into a complete theory of temporal summation in audition.

\section{References}

Aiba, T. S., \& Stevens, S. S. Relation of brightness to duration under light- and dark-adaptation. Vision. Res., 1964, 4, 391-401. Bills, M. A. The lag of visual sensation in its relation to wavelength and intensity of light. Psychol. Monogr., 1920, 28, No. 5.
Ekman, G. Two generalized ratio scaling methods. J. Psychol. $1958,45,287-295$.

Ekman, G., Bergl und, B., \& Berglund, U. Loudness as a function of the duration of auditory stimulation. Rep., Psychol. Lab. Univ. Stockholm, 1966, No. 205.

Garner, W. R. Effect of frequency spectrum on temporal integration in the ear. J. Acoust. Soc. Amer., 1947, 19, 808-815.

Gerbrands, R., \& Stevens, J.C. A high-intensity flash source. Amer. J. Psychol., 1964, 77, 643-646.

Luce, R. D. On the possible psychophysical laws. Psychol. Rev., $1959,66,81-95$.

Marks, L. E. Brightness as a function of retinal locus. Percept \& Psychophys., (in press).

Miller, G. A. Perception of short bursts of noise. J. Acoust. Soc. Amer., 1948, 20, 160-170.

Raab. D. Magnitude estimation of the brightness of brief foveal stimuli. Science, $1962,135,42-43$.

Small, A. M., Jr., Brandt, J. F., \& Cox, P. G. Loudness as a function of signal duration. J. Acoust. Soc. Amer., 1962, 34, 513-514.

Stevens, J. C., \& Stevens, S. S. Physiological zero and the psychophysical law. In Proc. 16th Int. Congr. Psychol., Amsterdam: North Holland, 1961. Pp. 192-193.

Stevens, J. C., \& Stevens, S. S. Brightness function: Effect of adaptation. J. Opt. Soc. Amer., 1963, 53, 375-385.

Stevens, S. S. To honor Fechner and repeal his law. Science, 1961, $133,80-86$.

Stevens, S. S. Matching functions between loudness and ten other continua. Percept. \& Psychophys., 1966a, 1, 5-8.

Stevens, S. S. Duration, luminance, and the brightness exponent. Percept. \& Psychophys., 1966b, 1, 96-100.

Wright, H. N. Loudness as a function of duration. J. Acoust. Soc. Amer., 1965, 37, 1174. (Abstract)

Yntema, D. B. The probability of hearing a short tone near threshold. Unpublished doctoral dissertation, Harvard University, 1955.

Zwislocki, J. Theory of temporal auditory summation J. Acoust.. Soc. Amer., 1960, 32, 1046-1060.

\section{Note}

1. This research was supported in part by National Institutes of Health Grant NB-02974 and in part by National Science Foundation Grant GB-3211 (Laboratory of Psychophysics Report PPR-327).

(Accepted for publication August 1, 1966.) 